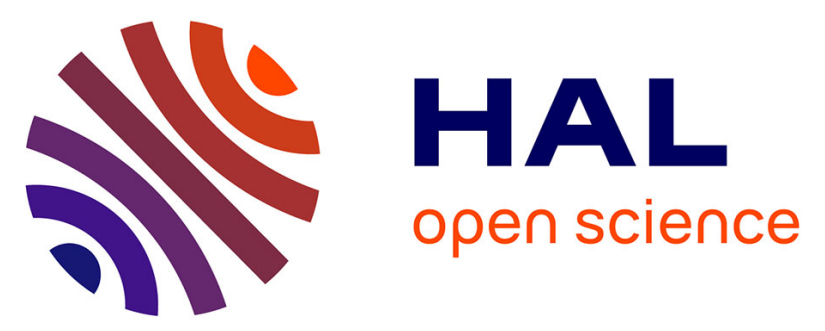

\title{
Exact and Fundamental Solution for an Anti-plane Crack Vertical to the Boundaries of a Magnetoelectroelastic Strip
}

\author{
Bao-Lin Wang, Yiu-Wing Mai
}

\section{- To cite this version:}

Bao-Lin Wang, Yiu-Wing Mai. Exact and Fundamental Solution for an Anti-plane Crack Vertical to the Boundaries of a Magnetoelectroelastic Strip. International Journal of Damage Mechanics, 2007, 16 (1), pp.77-94. 10.1177/1056789507060781 . hal-00571167

\section{HAL Id: hal-00571167 \\ https://hal.science/hal-00571167}

Submitted on 1 Mar 2011

HAL is a multi-disciplinary open access archive for the deposit and dissemination of scientific research documents, whether they are published or not. The documents may come from teaching and research institutions in France or abroad, or from public or private research centers.
L'archive ouverte pluridisciplinaire HAL, est destinée au dépôt et à la diffusion de documents scientifiques de niveau recherche, publiés ou non, émanant des établissements d'enseignement et de recherche français ou étrangers, des laboratoires publics ou privés. 


\title{
Exact and Fundamental Solution for an Anti-plane Crack Vertical to the Boundaries of a Magnetoelectroelastic Strip
}

\author{
Bao-Lin WANG* AND Yiu-Wing MAI \\ Centre for Advanced Materials Technology (CAMT) \\ School of Aerospace, Mechanical and Mechatronic Engineering \\ The University of Sydney, Sydney, NSW 2006, Australia
}

\begin{abstract}
In this article, we develop a closed-form solution for a crack in a magnetoelectroelastic strip of finite width, subjected to anti-plane mechanical and in-plane electric and magnetic fields. Explicit expressions for the stresses, electric and magnetic fields, together with their intensity factors are obtained for the two extreme cases of an impermeable crack and a permeable crack. Solutions for some special cases, such as a center crack, an edge crack, a semi-infinite medium, and an infinite medium, are also obtained in closed-forms. The problem of two symmetric and collinear cracks is also discussed. It is found that the electrically and magnetically permeable conditions on the crack profile are important in obtaining the correct crack tip electromagnetic filed intensity factors. The stress intensity factor, however, does not depend on the crack electromagnetic boundary condition assumptions.
\end{abstract}

KEY WORDS: magnetoelectroelastic materials, fracture mechanics, cracks.

\section{INTRODUCTION}

Ll MATERIALS SUFFER damages when subjected to external loading
conditions (Aglan and Fateh, 2006; Alam and Jenkins, 2005; Bielski
et al., 2006; Lin et al., 2005; Lu et al., 2005; Voyadjis and Abu Al-Rub, 2006;
Wei et al., 2006; Ye et al., 2006). This is also true for magnetoelectroelastic

*Author to whom correspondence should be addressed. E-mail: baolin.wang@aeromech.usyd.edu.au, wangbl2001@ hotmail.com

International Journal of DAMAGE MeCHANICS, Vol. 16-January 2007 
materials where the application of either a magnetic field or an electrical field induces an electrical polarization as well as magnetization. That is, an electric field can be induced by a magnetic field and vice versa. Magnetoelectroelastic material has both theoretical and practical significance in solid-state physics, biomechanical engineering, and smart materials and devices. Defects are often unavoidable in such materials because of their brittle property. Thus, in recent years, the study of magnetoelectroelastic materials with defects has received considerable interest. For examples, two-dimensional (2D) Green functions for a magnetoelectroelastic anisotropic medium with an elliptical cavity or rigid inclusion (Chung and Ting, 1995), Green's functions for an elliptical cavity by taking into account the electric-magnetic fields inside the cavity (Liu et al., 2001), general solution of three-dimensional (3D) problems in magnetoelectroelastic media (Wang and Shen, 2002), the single and collinear permeable crack problems using an elliptical-cavity-based approach and a crack-based method (Gao et al., 2003a, b), closed-form expressions for the energy release rate of an impermeable or permeable crack in a piezomagnetic/piezoelectric solid (Wang and Mai, 2003, 2004), macrocrack-microcrack interaction problem (Tian and Gabbert, 2005a), interface crack problem (Tian and Gabbert, 2005b), moving crack problem ( $\mathrm{Hu}$ and $\mathrm{Li}$, 2005). Recently, Gao et al. (2004) developed a Stroh-type formalism for the mode III fracture mechanics of an elliptical cavity in an infinite magnetoelectroelastic solid.

In many cases, solutions in closed-form are desired for accurate analysis and design. Closed-form solutions for the crack tip fields are essential for material scientists and engineers for the damage evaluation of the electromagnetoelastic materials. Owing to the mathematical complexity, certain practical problems are only solved with recourse to numerical schemes, and it is difficult to obtain their analytical solutions in closed-form. In this article, a closed-form solution for a through mode-III crack in a magnetoelectroelastic strip is presented as follows: the 'Description of the Problem', section gives basic governing equations for a linear magnetoelectroelastic solid and the solution for anti-plane deformation. This solution is expressed in Fourier transform form with some unknown variables. These unknown variables are determined in the 'Closed-form Solution' section. The next four sections give solutions, respectively, for electrically impermeable and magnetically impermeable crack, electrically permeable and magnetically impermeable crack, electrically impermeable and magnetically permeable crack, electrically permeable and magnetically permeable crack. The 'Some Special Cases' section provides solutions for some special cases, including a center crack problem, edge crack problem and semi-infinite medium. Finally, some conclusions are drawn in the last section. 


\section{DESCRIPTION OF THE PROBLEM}

Consider a magnetoelectroelastic strip occupying $0 \leq x \leq h,-\infty<y<\infty$, $-\infty<z<\infty$, with a through Griffith crack of length $2 a$ located at the $y=0$ plane, as shown in Figure 1. Here Cartesian coordinates $x, y, z$ are the principal axes of the material symmetry while the $z$-axis is oriented in the poling direction of the magnetoelectroelastic layer. From the viewpoint of applications, anti-plane crack problems often provide a useful analog to the more interesting in-plane fracture problems. In this study, we consider the following anti-plane mechanical and in plane electromagnetic loads:

$$
\sigma_{y z}(x, \pm \infty)=\tau_{0}, \quad D_{y}(x, \pm \infty)=D_{0}, \quad B_{y}(x, \pm \infty)=B_{0},
$$

in which the subscript 0 represents the prescribed values. The governing equations for the magnetoelectroelastic medium whose poling direction is perpendicular to the $x-y$ plane are (Wang and Mai, 2004):

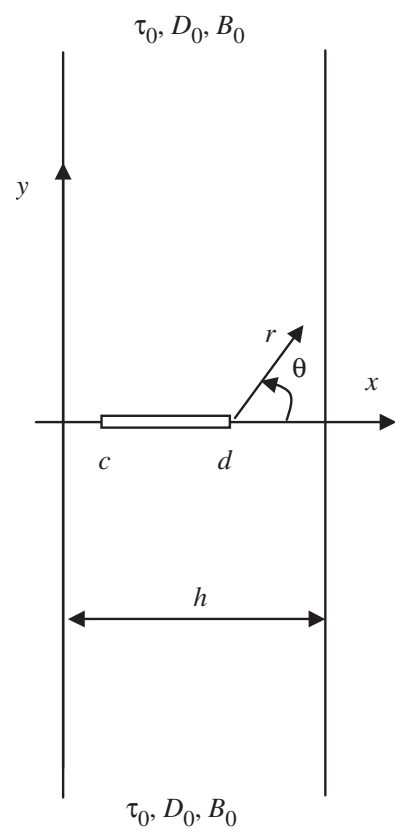

Figure 1. Geometry and coordinate of a crack vertical to the boundary of a magnetoelectroelastic strip (crack length $2 a=d-c$; for center crack problem, $c=h-d$; for edge crack problem, $d=h, c>0$; for semi-infinite medium, $h \rightarrow \infty$ and $c$ and $d$ are finite). 
- Constitutive equations:

$$
\begin{array}{ll}
\sigma_{x z}=c_{44} \frac{\partial w}{\partial x}+e_{15} \frac{\partial \phi}{\partial x}+h_{15} \frac{\partial \varphi}{\partial x}, & \sigma_{y z}=c_{44} \frac{\partial w}{\partial y}+e_{15} \frac{\partial \phi}{\partial y}+h_{15} \frac{\partial \varphi}{\partial y} \\
D_{x}=e_{15} \frac{\partial w}{\partial x}-\epsilon_{11} \frac{\partial \phi}{\partial x}-\beta_{11} \frac{\partial \varphi}{\partial x}, & D_{y}=e_{15} \frac{\partial w}{\partial y}-\epsilon_{11} \frac{\partial \phi}{\partial y}-\beta_{11} \frac{\partial \varphi}{\partial y}, \\
B_{x}=h_{15} \frac{\partial w}{\partial x}-\beta_{11} \frac{\partial \phi}{\partial x}-\gamma_{11} \frac{\partial \varphi}{\partial x}, & B_{y}=h_{15} \frac{\partial w}{\partial y}-\beta_{11} \frac{\partial \phi}{\partial y}-\gamma_{11} \frac{\partial \varphi}{\partial y},
\end{array}
$$

and

- equilibrium equations (in the absence of body forces, distributed electric charges, and distributed magnetic source):

$$
\begin{aligned}
& c_{44} \nabla^{2} w+e_{15} \nabla^{2} \phi+h_{15} \nabla^{2} \varphi=0, \\
& e_{15} \nabla^{2} w-\epsilon_{11} \nabla^{2} \phi-\beta_{11} \nabla^{2} \varphi=0, \\
& h_{15} \nabla^{2} w-\beta_{11} \nabla^{2} \phi-\gamma_{11} \nabla^{2} \varphi=0 .
\end{aligned}
$$

In Equations (2) and (3), $w$ is the anti-plane mechanical deformation; $\phi$ and $\varphi$ are the electric potential and magnetic potential, respectively; $\sigma_{i j}, D_{i}$, and $B_{i}$ are components of stress, electrical displacement, and magnetic induction, respectively; $c_{44}, e_{15}, h_{15}$ and $\beta_{11}$ are elastic, piezoelectric, piezomagnetic, and electromagnetic constants, respectively; and $\epsilon_{11}$ and $\gamma_{11}$ are dielectric permeability and magnetic permeability, respectively.

Due to symmetry of the problem, there are additional elastic, electric, and magnetic continuity conditions along the crack line:

$$
w(x, 0)=0, \quad \phi(x, 0)=0, \quad \varphi(x, 0)=0, \quad x \notin(c, d),
$$

In solving the crack problem, the crack surfaces are usually stress free. However, since the medium inside the crack (usually air or vacuum) allows some penetration of the electric and magnetic fields, the electric and magnetic field inside the crack may not be zero. Suppose the normal components of electric displacement and magnetic induction inside the crack are $d_{0}$ and $b_{0}$, respectively, then on the crack surface we have:

$$
\sigma_{y z}(x, 0)=0, \quad D_{y}(x, 0)=d_{0}, \quad B_{y}(x, 0)=b_{0}, \quad x \in(c, d) .
$$

The quantities $d_{0}$ and $b_{0}$ are unknown and will be determined later. 
Due to symmetry, it suffices to analyze the upper-half portion of the strip, i.e., $0 \leq x \leq h, y \geq 0$. The magnetoelectroelastic field in the lower portion of the strip can be directly obtained by symmetry consideration. Analogous to the solution for elastic materials ( $\mathrm{Li}, 2003)$, it is can be shown that an appropriate solution of Equation (3), in connection with Equation (2), can be expressed as the following series:

$$
\left\{\begin{array}{l}
w(x, y) \\
\phi(x, y) \\
\varphi(x, y)
\end{array}\right\}=\sum_{n=1}^{\infty} \exp (-2 n b y) \cos (2 n b x)\left\{\begin{array}{l}
A_{n} \\
B_{n} \\
C_{n}
\end{array}\right\}+\left\{\begin{array}{l}
w_{0} \\
\phi_{0} \\
\varphi_{0}
\end{array}\right\} y, \quad 0 \leq x \leq h, \quad y \geq 0
$$

where $b=\pi / 2 h$ and $\left(A_{n}, B_{n}, C_{n}\right)$ are unknown functions to be determined from the prescribed conditions Equations (4) and (5) on the cracked plane. The quantities $w_{0}, \phi_{0}$, and $\varphi_{0}$ are determined from the far-field conditions in Equation (1):

$$
\left\{\begin{array}{l}
w_{0} \\
\phi_{0} \\
\varphi_{0}
\end{array}\right\}=\left[\begin{array}{ccc}
\bar{c}_{44} & \bar{e}_{15} & \bar{h}_{15} \\
\bar{e}_{15} & -\bar{\epsilon}_{11} & -\bar{\beta}_{11} \\
\bar{h}_{15} & -\bar{\beta}_{11} & -\bar{\gamma}_{11}
\end{array}\right]\left\{\begin{array}{c}
\tau_{0} \\
D_{0} \\
B_{0}
\end{array}\right\}
$$

where

$$
\left[\begin{array}{ccc}
\bar{c}_{44} & \bar{e}_{15} & \bar{h}_{15} \\
\bar{e}_{15} & -\bar{\epsilon}_{11} & -\bar{\beta}_{11} \\
\bar{h}_{15} & -\bar{\beta}_{11} & -\bar{\gamma}_{11}
\end{array}\right]=\left[\begin{array}{ccc}
c_{44} & e_{15} & h_{15} \\
e_{15} & -\epsilon_{11} & -\beta_{11} \\
h_{15} & -\beta_{11} & -\gamma_{11}
\end{array}\right]^{-1}
$$

Equation (6) satisfies the free boundary conditions on the $x=0$ and $x=h$ planes, that is $\sigma_{x z}(0, y)=0, \quad D_{x}(0, y)=0, \quad B_{x}(0, y)=0, \quad \sigma_{x z}(h, y)=0$, $D_{x}(h, y)=0, B_{x}(h, y)=0$.

From the constitutive Equations (2), the stresses, electric displacements, and magnetic inductions can be expressed in terms of $A_{n}, B_{n}$, and $C_{n}$. For example, we have

$$
\begin{aligned}
\left\{\begin{array}{c}
\sigma_{y z}(x, y) \\
D_{y}(x, y) \\
B_{y}(x, y)
\end{array}\right\}= & \left\{\begin{array}{c}
\tau_{0} \\
D_{0} \\
B_{0}
\end{array}\right\}-2 b\left[\begin{array}{ccc}
c_{44} & e_{15} & h_{15} \\
e_{15} & -\epsilon_{11} & -\beta_{11} \\
h_{15} & -\beta_{11} & -\gamma_{11}
\end{array}\right] \\
& \times \sum_{n=1}^{\infty} n \exp (-2 n b y) \cos (2 n b x)\left\{\begin{array}{c}
A_{n} \\
B_{n} \\
C_{n}
\end{array}\right\},
\end{aligned}
$$


In which the unknowns $A_{n}, B_{n}$, and $C_{n}$ will be determined from the mixed mode boundary conditions on the cracked plane in the following section.

\section{CLOSED-FORM SOLUTION}

In view of Equations (6) and (9), using the boundary conditions, Equations (4) and (5) yield simultaneous triple series equations for $A_{n}, B_{n}$, and $C_{n}$,

$$
\begin{aligned}
& \sum_{n=1}^{\infty} \cos (2 n b x)\left\{\begin{array}{l}
A_{n} \\
B_{n} \\
C_{n}
\end{array}\right\}=0, \quad x \notin(c, d), \\
& \sum_{n=1}^{\infty} n \cos (2 n b x)\left\{\begin{array}{l}
A_{n} \\
B_{n} \\
C_{n}
\end{array}\right\}=\frac{1}{2 b}\left[\begin{array}{ccc}
\bar{c}_{44} & \bar{e}_{15} & \bar{h}_{15} \\
\bar{e}_{15} & -\bar{\epsilon}_{11} & -\bar{\beta}_{11} \\
\bar{h}_{15} & -\bar{\beta}_{11} & -\bar{\gamma}_{11}
\end{array}\right]\left\{\begin{array}{c}
\tau_{0} \\
D_{0}-d_{0} \\
B_{0}-b_{0}
\end{array}\right\}, x \in(c, d) .
\end{aligned}
$$

If we define the generalized screw dislocation density factions $w(x), \phi(x)$, and $\varphi(x)$ :

$$
w(x)=\frac{\partial w(x, 0)}{\partial x}, \quad \phi(x)=\frac{\partial \phi(x, 0)}{\partial x}, \quad \varphi(x)=\frac{\partial \varphi(x, 0)}{\partial x}
$$

then $A_{n}, B_{n}$, and $C_{n}$ can be expressed as:

$$
\left\{\begin{array}{l}
A_{n} \\
B_{n} \\
C_{n}
\end{array}\right\}=-\frac{1}{n b h} \int_{c}^{d} \sin (2 n b s)\left\{\begin{array}{l}
w(s) \\
\phi(s) \\
\varphi(s)
\end{array}\right\} \mathrm{d} s
$$

Substituting Equation (13) into Equation (11), a system of singular integral equations for $w(x), \phi(x)$, and $\varphi(x)$ is obtained as

$$
\begin{aligned}
& \frac{1}{h} \int_{c}^{d} \frac{\sin (2 b s)}{\cos (2 b s)-\cos (2 b x)}\left\{\begin{array}{l}
w(s) \\
\phi(s) \\
\varphi(s)
\end{array}\right\} \mathrm{d} s=\left[\begin{array}{ccc}
\bar{c}_{44} & \bar{e}_{15} & \bar{h}_{15} \\
\bar{e}_{15} & -\bar{\epsilon}_{11} & -\bar{\beta}_{11} \\
\bar{h}_{15} & -\bar{\beta}_{11} & -\bar{\gamma}_{11}
\end{array}\right]\left\{\begin{array}{c}
\tau_{0} \\
D_{0}-d_{0} \\
B_{0}-b_{0}
\end{array}\right\}, \\
& x \in(c, d)
\end{aligned}
$$

In obtaining Equation (14), the following identity has been used:

$$
\sum_{n=1}^{\infty} \frac{2}{n} \cos (n \vartheta) \cos (n \theta)=-\ln (2|\cos (\vartheta)-\cos (\theta)|) .
$$


In what follows, we define the symbols:

$$
s_{1}=\cos (2 b s), \quad x_{1}=\cos (2 b x), \quad c_{1}=\cos (2 b c), \quad d_{1}=\cos (2 b d) .
$$

Using Equation (16), Equation (14) can be rewritten as:

$$
\frac{1}{\pi} \int_{d_{1}}^{c_{1}} \frac{1}{s_{1}-x_{1}}\left\{\begin{array}{l}
w_{1}\left(s_{1}\right) \\
\phi_{1}\left(s_{1}\right) \\
\varphi_{1}\left(s_{1}\right)
\end{array}\right\} \mathrm{d} s_{1}=\left[\begin{array}{ccc}
\bar{c}_{44} & \bar{e}_{15} & \bar{h}_{15} \\
\bar{e}_{15} & -\bar{\epsilon}_{11} & -\bar{\beta}_{11} \\
\bar{h}_{15} & -\bar{\beta}_{11} & -\bar{\gamma}_{11}
\end{array}\right]\left\{\begin{array}{c}
\tau_{0} \\
D_{0}-d_{0} \\
B_{0}-b_{0}
\end{array}\right\}, \quad x_{1} \in\left(d_{1}, c_{1}\right),
$$

in which the notations $w_{1}\left(s_{1}\right)=w(s), \phi_{1}\left(s_{1}\right)=\phi(s), \varphi_{1}\left(s_{1}\right)=\varphi(s)$ have been adopted. Equation (17) is a system of singular integral equations with Cauchy kernel. By using the techniques described in Muskhelisvili (1953), the solution of Equation (17) is found to be:

$$
\begin{aligned}
\left\{\begin{array}{l}
w_{1}\left(s_{1}\right) \\
\phi_{1}\left(s_{1}\right) \\
\varphi_{1}\left(s_{1}\right)
\end{array}\right\}= & \frac{1}{2}\left[\begin{array}{ccc}
\bar{c}_{44} & \bar{e}_{15} & \bar{h}_{15} \\
\bar{e}_{15} & -\bar{\epsilon}_{11} & -\bar{\beta}_{11} \\
\bar{h}_{15} & -\bar{\beta}_{11} & -\bar{\gamma}_{11}
\end{array}\right]\left[\sqrt{\frac{s_{1}-d_{1}}{c_{1}-s_{1}}}-\sqrt{\frac{c_{1}-s_{1}}{s_{1}-d_{1}}}\right]\left\{\begin{array}{c}
\tau_{0} \\
D_{0}-d_{0} \\
B_{0}-b_{0}
\end{array}\right\} \\
& +\frac{1}{\sqrt{\left(c_{1}-s_{1}\right)\left(s_{1}-d_{1}\right)}}\{C\},
\end{aligned}
$$

where $\{C\}$ is a constant column of three elements, which can be determined for the problem under consideration by requiring that

$$
\int_{c}^{d}\left\{\begin{array}{l}
w(s) \\
\phi(s) \\
\varphi(s)
\end{array}\right\} \mathrm{d} s=0
$$

Following the methods of $\mathrm{Li}$ (2003) and after some manipulations, we get

$$
\left\{\begin{array}{l}
w_{1}\left(s_{1}\right) \\
\phi_{1}\left(s_{1}\right) \\
\varphi_{1}\left(s_{1}\right)
\end{array}\right\}=\left[\begin{array}{ccc}
\bar{c}_{44} & \bar{e}_{15} & \bar{h}_{15} \\
\bar{e}_{15} & -\bar{\epsilon}_{11} & -\bar{\beta}_{11} \\
\bar{h}_{15} & -\bar{\beta}_{11} & -\bar{\gamma}_{11}
\end{array}\right] \frac{\left(s_{1}+1\right)-\left(d_{1}+1\right) \chi}{\sqrt{\left(c_{1}-s_{1}\right)\left(s_{1}-d_{1}\right)}}\left\{\begin{array}{c}
\tau_{0} \\
D_{0}-d_{0} \\
B_{0}-b_{0}
\end{array}\right\},
$$


with

$$
\chi=\frac{\Pi(\xi, k)}{K(k)},
$$

where $K(k)$ and $\pi(\xi, k)$ are the complete elliptical integrals of the first and third kinds, respectively, viz

$$
\begin{aligned}
K(k) & =\int_{0}^{\pi / 2} \frac{1}{\sqrt{1-k^{2} \sin ^{2}(\theta)}} \mathrm{d} \theta, \\
\Pi(\xi, k) & =\int_{0}^{\pi / 2} \frac{1}{\left[1+\xi \sin ^{2}(\theta)\right] \sqrt{1-k^{2} \sin ^{2}(\theta)}} \mathrm{d} \theta,
\end{aligned}
$$

with

$$
k=\frac{\sqrt{\tan ^{2}(b d)-\tan ^{2}(b c)}}{\tan (b d)}, \quad \xi=\frac{\tan ^{2}(b c)-\tan ^{2}(b d)}{\sec ^{2}(b d)} .
$$

Now substituting Equation (20) into Equation (13), then into Equation (9), we obtain the explicit expressions for the anti-plane shear stress, in-plane electric displacement, and magnetic induction along the crack line in terms of the original variables. The results are very complicated functions of $x$. Near the crack tips these quantities are:

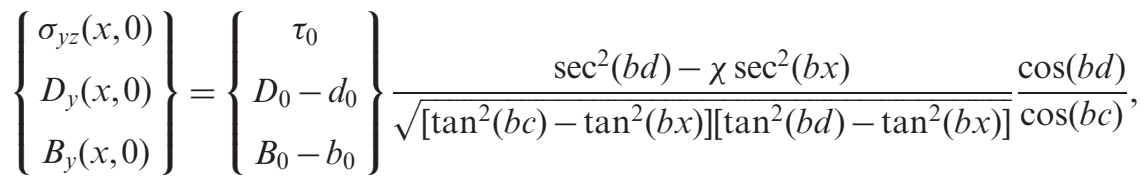

for $x \rightarrow c-0$, and

$$
\left\{\begin{array}{c}
\sigma_{y z}(x, 0) \\
D_{y}(x, 0) \\
B_{y}(x, 0)
\end{array}\right\}=\left\{\begin{array}{c}
\tau_{0} \\
D_{0}-d_{0} \\
B_{0}-b_{0}
\end{array}\right\} \frac{\chi \sec ^{2}(b x)-\sec ^{2}(b d)}{\sqrt{\left[\tan ^{2}(b x)-\tan ^{2}(b c)\right]\left[\tan ^{2}(b x)-\tan ^{2}(b c)\right]}} \frac{\cos (b d)}{\cos (b d)},
$$

for $x \rightarrow d+0$. As expected, these quantities exhibit the usual square-root singularity near the crack tip. 
The displacement $w(x, 0)$, electric potential $\phi(x, 0)$, and magnetic potential $\varphi(x, 0)$ on the upper surface of the crack can be obtained in closed form as well. With the solution of Equation (20), integrating according to the original variables between the limits $c$ and $d(c \leq x \leq d)$ yields the following:

$$
\begin{aligned}
\left\{\begin{array}{l}
w(x, 0) \\
\phi(x, 0) \\
\varphi(x, 0)
\end{array}\right\}= & \frac{2 h}{\pi}\left[\begin{array}{ccc}
\bar{c}_{44} & \bar{e}_{15} & \bar{h}_{15} \\
\bar{e}_{15} & -\bar{\epsilon}_{11} & -\bar{\beta}_{11} \\
\bar{h}_{15} & -\bar{\beta}_{11} & -\bar{\gamma}_{11}
\end{array}\right]\left\{\begin{array}{c}
\tau_{0} \\
D_{0}-d_{0} \\
B_{0}-b_{0}
\end{array}\right\} \\
& \times \frac{\cos (b d)}{\tan (b d) \cos (b c)}[\chi F(\varpi, k)-\Pi(\varpi, \xi, k)],
\end{aligned}
$$

where $F(\varpi, k)$ and $\Pi(\varpi, \xi, k)$ are the incomplete elliptical integrals of the first and the third kinds, respectively, with

$$
\varpi=\sin ^{-1}\left(\sqrt{\frac{\tan ^{2}(b d)-\tan ^{2}(b x)}{\tan ^{2}(b d)-\tan ^{2}(b c)}}\right),
$$

and $k$ and $\xi$ being given in Equation (23).

Of particular interest is the crack tip field from the viewpoint of the fracture mechanics. It is desirable to determine the intensity factors of the magnetoelectroelastic field at the crack tip. From the definitions of the mode-III stress intensity factor $K_{\text {III }}$, the electric displacement intensity factor $K_{\mathrm{D}}$, and the magnetic induction intensity factor $K_{\mathrm{B}}$ :

$$
\begin{gathered}
\left\{\begin{array}{c}
K_{\mathrm{III}}(c) \\
K_{\mathrm{D}}(c) \\
K_{\mathrm{B}}(c)
\end{array}\right\}=\lim _{x \rightarrow c-0}\left\{\begin{array}{c}
\sigma_{y z}(x, 0) \\
D_{y}(x, 0) \\
B_{y}(x, 0)
\end{array}\right\} \sqrt{2 \pi(c-x)}, \\
\left\{\begin{array}{c}
K_{\mathrm{III}}(d) \\
K_{\mathrm{D}}(d) \\
K_{\mathrm{B}}(d)
\end{array}\right\}=\lim _{x \rightarrow d+0}\left\{\begin{array}{c}
\sigma_{y z}(x, 0) \\
D_{y}(x, 0) \\
B_{y}(x, 0)
\end{array}\right\} \sqrt{2 \pi(x-d)}
\end{gathered}
$$

we arrive at

$$
\left\{\begin{array}{c}
K_{\mathrm{III}}(x) \\
K_{\mathrm{D}}(x) \\
K_{\mathrm{B}}(x)
\end{array}\right\}=\left\{\begin{array}{c}
\tau_{0} \\
D_{0}-d_{0} \\
B_{0}-b_{0}
\end{array}\right\} f_{x}, \quad x=c \text { or } d,
$$


in which the functions $f_{c}$ and $f_{d}$ are solely determined from the geometry of the medium:

$$
\begin{aligned}
& f_{c}(c, d, h)=\sqrt{2 h \tan \left(\frac{\pi c}{2 h}\right)} \frac{\cos ^{2}(\pi c / 2 h)-\chi \cos ^{2}(\pi d / 2 h)}{\sin (\pi c / 2 h) \sqrt{\cos ^{2}(\pi c / 2 h)-\cos ^{2}(\pi d / 2 h)}}, \\
& f_{d}(c, d, h)=\sqrt{2 h \cot \left(\frac{\pi d}{2 h}\right)} \frac{\cos (\pi d / 2 h)[\chi-1]}{\sqrt{\cos ^{2}(\pi c / 2 h)-\cos ^{2}(\pi d / 2 h)}}
\end{aligned}
$$

The angular distributions of the stresses, electric displacements and magnetic inductions near the crack tip are related to the field intensity factors through (Wang and Mai, 2004):

$$
\begin{aligned}
\sigma_{x z} & =-\frac{K_{\mathrm{III}}}{\sqrt{2 \pi r}} \sin \frac{\theta}{2}, & \sigma_{y z} & =\frac{K_{\mathrm{III}}}{\sqrt{2 \pi r}} \cos \frac{\theta}{2}, \\
D_{x} & =-\frac{K_{\mathrm{D}}}{\sqrt{2 \pi r}} \sin \frac{\theta}{2}, & D_{y} & =\frac{K_{\mathrm{D}}}{\sqrt{2 \pi r}} \cos \frac{\theta}{2}, \\
B_{x} & =-\frac{K_{\mathrm{B}}}{\sqrt{2 \pi r}} \sin \frac{\theta}{2}, & B_{y} & =\frac{K_{\mathrm{B}}}{\sqrt{2 \pi r}} \cos \frac{\theta}{2},
\end{aligned}
$$

in which $r$ and $\theta$ are shown in Figure 1. The energy release rate can be obtained from the virtual crack closure integral (Wang and Mai, 2004):

$$
\begin{aligned}
G=\lim _{\delta \rightarrow 0} \frac{1}{\delta} \int_{0}^{\delta}[ & \sigma_{y z}(x+a, 0) w(x+a-\delta, 0) \\
& +D_{y}(x+a, 0) \phi(x+a-\delta, 0) \\
& \left.+B_{y}(x+a, 0) \varphi(x+a-\delta, 0)\right] \mathrm{d} x .
\end{aligned}
$$

From Equations (25) and (29), an expression relating $G$ to $\left(K_{\mathrm{III}}, K_{\mathrm{D}}, K_{\mathrm{B}}\right)$ can be obtained:

$$
G=\frac{1}{2}\left(K_{\mathrm{III}}, K_{\mathrm{D}}, K_{\mathrm{B}}\right)\left(\begin{array}{ccc}
c_{44} & e_{15} & h_{15} \\
e_{15} & -\epsilon_{11} & -\beta_{11} \\
h_{15} & -\beta_{11} & -\gamma_{11}
\end{array}\right)^{-1}\left\{\begin{array}{c}
K_{\mathrm{III}} \\
K_{\mathrm{D}} \\
K_{\mathrm{B}}
\end{array}\right\}
$$

It can be seen from Equation (28) that the stress intensity factor $K_{\text {III }}$ does not depend on the material properties and is the same as the elastic materials given by $\mathrm{Li}(2003)$. $K_{\mathrm{III}}$ is solely determined by the geometry of the problem. 
Now, the crack tip field intensity factors, the angular distributions of the stresses, electric displacements and magnetic inductions, as well as the energy release rate, have been obtained analytically in terms of the applied $\left(\tau_{0}, D_{0}, B_{0}\right)$ at the far-field and $\left(d_{0}, b_{0}\right)$ on the crack faces. Since $\left(d_{0}, b_{0}\right)$ remains unknown, additional assumptions are needed to obtain the full solution. It is well known that in piezoelectric fracture, the electrically impermeable and permeable crack assumptions are usually adopted. Analogously, the crack can be assumed to be magnetically impermeable or permeable in magnetoelectroelastic fracture. In the next four sections, the electrically impermeable and magnetically impermeable crack, the electrically permeable and magnetically impermeable crack, the electrically impermeable and magnetically permeable crack, the electrically permeable and magnetically permeable crack assumptions will be investigated separately. In these sections, the crack is assumed to be a very thin notch of infinitesimal height.

\section{THE ELECTRICALLY IMPERMEABLE AND MAGNETICALLY IMPERMEABLE CRACK ASSUMPTION (FULLY IMPERMEABLE CRACK)}

For such an assumption, the crack is absolutely insulated to electric and magnetic fields. Hence, the normal components of the electric displacement vector and the magnetic induction vector vanish everywhere inside the crack, that is, $d_{0}=0$ and $b_{0}=0$. Then the field intensity factors can be obtained directly from Equation (28) as follows:

$$
\left\{\begin{array}{c}
K_{\mathrm{III}}(c) \\
K_{\mathrm{D}}(c) \\
K_{\mathrm{B}}(c)
\end{array}\right\}=\left\{\begin{array}{c}
\tau_{0} \\
D_{0} \\
B_{0}
\end{array}\right\} f_{c}, \quad\left\{\begin{array}{c}
K_{\mathrm{III}}(d) \\
K_{\mathrm{D}}(d) \\
K_{\mathrm{B}}(d)
\end{array}\right\}=\left\{\begin{array}{c}
\tau_{0} \\
D_{0} \\
B_{0}
\end{array}\right\} f_{d} .
$$

Solutions near the crack tip and the energy release rate are obtained from Equations (30) and (32) with the substitution of Equation (33). It is clear that for an electrically and magnetically impermeable crack, the material properties do not enter into the field intensity factors.

\section{THE ELECTRICALLY PERMEABLE AND MAGNETICALLY IMPERMEABLE CRACK ASSUMPTION}

For such an assumption, the upper and lower surfaces of the crack are electrically contacted but magnetically insulated. Hence, the electric 
potential $\phi(x, 0)$ and the normal component of the magnetic induction vector $b_{0}$ vanish everywhere inside the crack, i.e., $\phi(x)=0$ and $b_{0}=0$. Therefore, the electric displacement inside the crack $d_{0}$ can be obtained from Equation (25) giving:

$$
d_{0}=D_{0}-\frac{\bar{e}_{15}}{\bar{\epsilon}_{11}} \tau_{0}+\frac{\bar{\beta}_{11}}{\bar{\epsilon}_{11}} B_{0} .
$$

Substituting Equation (34) into Equation (28) yields the field intensity factors:

$$
\begin{aligned}
\left\{\begin{array}{c}
K_{\mathrm{III}}(c) \\
K_{\mathrm{B}}(c)
\end{array}\right\} & =\left\{\begin{array}{c}
\tau_{0} \\
B_{0}
\end{array}\right\} f_{c}, \quad\left\{\begin{array}{c}
K_{\mathrm{III}}(d) \\
K_{\mathrm{B}}(d)
\end{array}\right\}=\left\{\begin{array}{c}
\tau_{0} \\
B_{0}
\end{array}\right\} f_{d}, \\
K_{\mathrm{D}} & =\left(\frac{\bar{e}_{15}}{\bar{\epsilon}_{11}} K_{\mathrm{III}}-\frac{\bar{\beta}_{11}}{\bar{\epsilon}_{11}} K_{\mathrm{B}}\right) \\
& =\frac{e_{15} \gamma_{11}-\beta_{11} h_{15}}{c_{44} \gamma_{11}+h_{15}^{2}} K_{\mathrm{III}}+\frac{c_{44} \beta_{11}+e_{15} h_{15}}{c_{44} \gamma_{11}+h_{15}^{2}} K_{\mathrm{B}} .
\end{aligned}
$$

Solutions near the crack tip and the energy release rate are obtained from Equations (30) and (32) with the substitution of Equations (35) and (36). From Equation (36) we known that the electric displacement intensity factor and the energy release rate for the electrically permeable and magnetically impermeable crack can be expressed in terms of the applied stress and magnetic induction intensity factors.

\section{THE ELECTRICALLY IMPERMEABLE AND MAGNETICALLY PERMEABLE CRACK ASSUMPTION}

For such an assumption, the upper and lower surfaces of the crack are magnetically contacted but electrically insulated. Hence, the magnetic potential $\varphi(x, 0)$ and the normal component of the electric displacement vector $d_{0}$ vanish everywhere inside the crack, i.e., $\varphi(x)=0$ and $d_{0}=0$. Therefore, the magnetic induction inside the crack $b_{0}$ can be obtained from Equation (25) giving:

$$
b_{0}=B_{0}-\frac{\bar{h}_{15}}{\bar{\gamma}_{11}} \tau_{0}+\frac{\bar{\beta}_{11}}{\bar{\gamma}_{11}} D_{0}
$$


Substitution of Equation (37) into Equation (28) yields the field intensity factors:

$$
\begin{aligned}
\left\{\begin{array}{c}
K_{\mathrm{III}}(c) \\
K_{\mathrm{D}}(c)
\end{array}\right\} & =\left\{\begin{array}{c}
\tau_{0} \\
D_{0}
\end{array}\right\} f_{c}, \quad\left\{\begin{array}{c}
K_{\mathrm{III}}(d) \\
K_{\mathrm{D}}(d)
\end{array}\right\}=\left\{\begin{array}{c}
\tau_{0} \\
D_{0}
\end{array}\right\} f_{d}, \\
K_{\mathrm{B}} & =\left(\frac{\bar{h}_{15}}{\bar{\gamma}_{11}} K_{\mathrm{III}}-\frac{\bar{\beta}_{11}}{\bar{\gamma}_{11}} K_{\mathrm{D}}\right) \\
& =\frac{h_{15} \epsilon_{11}-\beta_{11} e_{15}}{c_{44} \epsilon_{11}+e_{15}^{2}} K_{\mathrm{III}}+\frac{c_{44} \beta_{11}+e_{15} h_{15}}{c_{44} \epsilon_{11}+e_{15}^{2}} K_{\mathrm{D}}
\end{aligned}
$$

Solutions near the crack tip and the energy release rate are obtained from Equations (30) and (32) with the substitution of Equations (38) and (39). It can be shown from Equation (39) that the magnetic induction intensity factor and the energy release rate for the electrically impermeable and magnetically permeable crack can be expressed in terms of the applied stress and electric displacement intensity factors.

\section{THE ELECTRICALLY PERMEABLE AND MAGNETICALLY PERMEABLE CRACK ASSUMPTION (FULLY PERMEABLE CRACK)}

For such an assumption, the upper and lower surfaces of the crack are electrically and magnetically contacted. Hence, the electric potential $\phi(x, 0)$ and the magnetic potential $\varphi(x, 0)$ vanish everywhere on the crack faces, i.e., $\phi(x)=0$ and $\varphi(x)=0$. Therefore, the electric displacement and the magnetic induction inside the crack $d_{0}$ and $b_{0}$ can be obtained from Equation (25). As a result, we obtain

$$
d_{0}=D_{0}-\frac{e_{15}}{c_{44}} \tau_{0}, \quad b_{0}=B_{0}-\frac{h_{15}}{c_{44}} \tau_{0},
$$

It then follows from Equation (28) that

$$
\begin{aligned}
K_{\mathrm{III}}(c) & =\tau_{0} f_{c}, \quad K_{\mathrm{III}}(d)=\tau_{0} f_{d}, \\
K_{\mathrm{D}} & =\frac{e_{15}}{c_{44}} K_{\mathrm{III}}, \quad K_{\mathrm{B}}=\frac{h_{15}}{c_{44}} K_{\mathrm{III}} .
\end{aligned}
$$

Solutions near the crack tip and the energy release rate are obtained from Equations (30) and (32) with the substitution of Equations (41) and (42). It is clear that for an electrically permeable and magnetically permeable crack assumption, the crack tip field intensity factors and the energy release rate are solely determined in terms of the applied stress intensity factor. 


\section{SOME SPECIAL CASES}

The closed-form expressions are given for a crack in a magnetoelectroelastic strip of finite width. It is possible to obtain the solutions for some special cases. We discuss this below.

\section{A Central Crack Normal to the Strip Boundaries}

In this case, the constant $c$ in the above expressions should be chosen such that $c=h-d$, which gives $\chi=1 /[1-\sin (\pi a / h)]$, where $a=(h-2 c) / 2$ is the half crack length. As a result, the functions $f_{c}$ and $f_{d}$ reduce to

$$
f_{c}=f_{d}=\sqrt{h \tan \left(\frac{\pi a}{h}\right)} .
$$

Solutions for the crack tips ( $x=c$ and $x=d$ ) are returned by replacing the quantities $f_{c}$ and $f_{d}$ in the five previous sections with Equation (43).

\section{One Crack Tip Approaches the Strip Boundary}

For this case, we consider $d \rightarrow h, c>0$. The function $f_{c}$ in this case reduces to

$$
f_{c}=\sqrt{2 h \cot \left(\frac{\pi c}{2 h}\right)}
$$

Solutions for the inner crack tip $(x=c)$ are returned by replacing the quantity $f_{c}$ in the previous five main sections with Equation (44). Note that this solution is for $d \rightarrow h$ so that the crack tip at $x=d$ is still closed (connected). For the case of $d=h$ the crack is edged and is discussed below.

\section{An Edge Crack Normal to the Strip Boundaries}

For an edge crack, we let $d=h$, and replace $c$ with $h-c>0$ (Figure 2(a)). From symmetry consideration, it is clear that the problem of an edge crack of length $c$ shown in Figure 2(a) is equivalent to the center crack of half-length $c$ shown in Figure 2(b). The function $f_{c}$ in this case can be obtained from the solution in the "A Central Crack Normal to the Strip Boundaries' section. The result is

$$
f_{c}=\sqrt{2 h \tan \left(\frac{\pi c}{2 h}\right)} .
$$


$\tau_{0}, D_{0}, B_{0}$

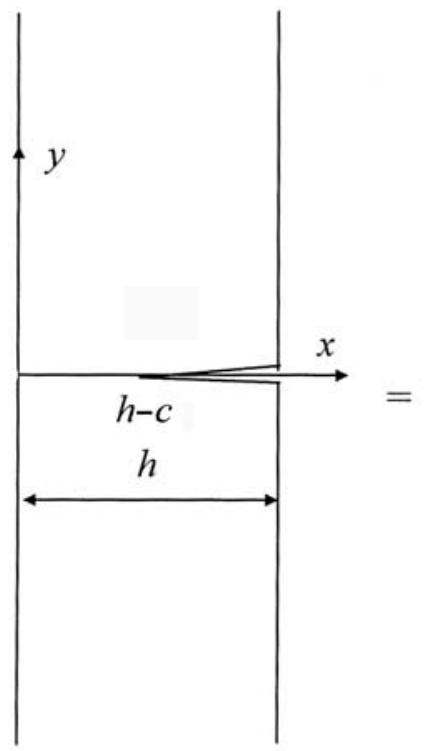

(a) $\tau_{0}, D_{0}, B_{0}$

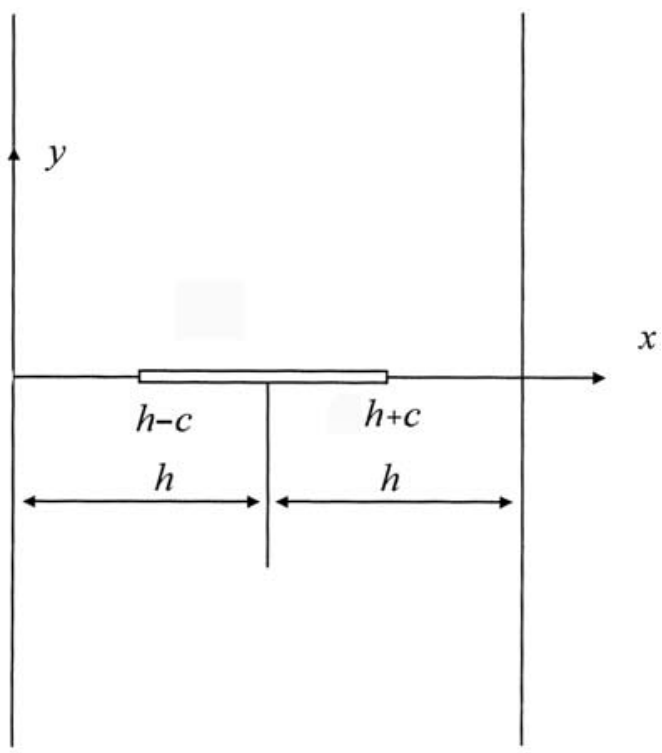

(b)

Figure 2. Equivalence of the edge crack problem (a) and the center crack problem (b).

Therefore, solutions for the inner tip of the edge crack $(x=c)$ shown in Figure 2(a) are returned by replacing the quantity $f_{c}$ in the five previous main sections with Equation (45). Different from in the case of the 'One Crack Tip Approaches the Strip Boundary' section, the crack tip $x=d$ (or $x=h)$ is disconnected for the present edge crack problem.

\section{Semi-infinite Medium}

A mode-III crack in a semi-infinite medium can be taken as the limiting case of the present study as $h \rightarrow \infty$. In fact, by employing relations among the elliptical integrals of the first, the second, and the third kind, and setting $h \rightarrow \infty$, a direct evaluation from Equations (29) leads to:

$$
\begin{aligned}
& f_{c}(c, d, h)=\sqrt{\pi c} \frac{\lambda-(c / d)^{2}}{(c / d) \sqrt{1-(c / d)^{2}}}, \\
& f_{d}(c, d, h)=\sqrt{\pi d} \frac{1-\lambda}{(c / d) \sqrt{1-(c / d)^{2}}},
\end{aligned}
$$


where

$$
\lambda=\frac{E\left(\sqrt{1-(c / d)^{2}}\right)}{K\left(\sqrt{1-(c / d)^{2}}\right)},
$$

in which $E$ and $K$ are the complete elliptical integrals of the first, and the second kind, respectively.

\section{CONCLUSIONS}

Exact and fundamental solution has been obtained in closed-form for a crack in a magnetoelectroelastic strip of finite width. Expressions for the crack tip field intensity factors, the electromagnetic fields inside the crack are given. The electrically and magnetically impermeable and permeable crack assumptions are investigated. This article is an extension of the work by Li (2003), which considered the pure elasticity materials. The work of Gao et al. (2004) also gave the solution to the magnetoelectroelastic effect but only the solution for infinite media are obtained.

It is found that applications of electric and magnetic fields do not change the stress intensity factors. The values of the stress intensity factor are identical for any kind of crack electric and magnetic boundary condition assumptions (i.e., the crack face electric and magnetic boundary conditions have no effect on the stress intensity factor). The result of the stress intensity factor for magnetoelectroelastic materials is the same as the solution for the elastic materials given by $\mathrm{Li}$ (2003).

Although the analysis is restricted to the single crack problem, the solution for two symmetric collinear cracks (Figure 3) can be obtained directly from symmetry consideration. Obviously, the $x \geq 0$ part of Figure 3 has the same solution as that of Figure 1. Further closed-form expressions for two collinear cracks whose outer tips approach the boundaries of the magnetoelectroelastic strip are the same as those for the single crack problem given in the 'One Crack Tip Approaches the Strip Boundary' section, closed-form expressions for two collinear edge mode-III cracks of equal length $h-c$ and separated each other by a distance $2 c$ in a magnetoelectroelastic strip are the same as those for the single edge crack in a strip given in the 'An Edge Crack Normal to the Strip Boundaries' section. Closed-form expressions for two collinear mode-III cracks of equal length $d-c$ and separated from each other by a distance $2 c$ in an infinite magnetoelectroelastic medium are the same as those for the single crack in the semi-infinite medium given in the 'Semi-Infinite Medium' section. 


$$
\tau_{0}, D_{0}, B_{0}
$$

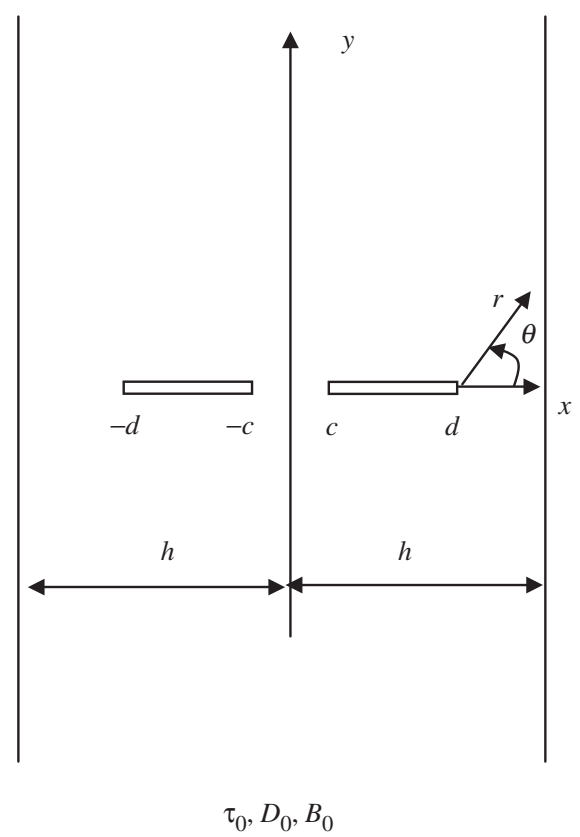

Figure 3. Two collinear cracks symmetrically located in a magnetoelectroelastic strip (crack length $2 a=d-c$; for two collinear edge cracks, $d=h, c>0$; for infinite medium with two collinear cracks, $h \rightarrow \infty$ and $c$ and $d$ are finite. Because of symmetry, the solution for two collinear cracks for $d \rightarrow h$ and for $d=h$ are the same as that for $d \rightarrow h$ and for $d=h$ for one single edge crack in Figure 1, and the solution for two collinear cracks in the infinite medium is the same as one single crack in a semi-infinite medium (in Figure 1 for $h \rightarrow \infty$ ).

\section{ACKNOWLEDGMENTS}

The authors would like to thank the Australian Research Council (ARC) for the continuing support of this research (projects \#DP0346037 and \#DP0665856). BLW and YWM are, respectively, Australian Research Fellow and Australian Federation Fellow supported by the ARC and tenable at the University of Sydney.

\section{REFERENCES}

Aglan, H.A. and Fateh, M. (2006). Fatigue Damage Tolerance of Bainitic and Pearlitic Rail Steels, International Journal of Damage Mechanics, 15(4): 393-410.

Alam, M.S. and Jenkins, C.H. (2005). Damage Tolerance in Naturally Compliant Structures, International Journal of Damage Mechanics, 14(4): 365-384. 
Bielski, J., Skrzypek, J.J. and Kuna-Ciskal, H. (2006). Implementation of a Model of Coupled Elastic-plastic Unilateral Damage Material to Finite Element Code, International Journal of Damage Mechanics, 15(1): 5-39.

Chung, M.Y. and Ting, T.C.T. (1995). The Green Function for a Piezoelectric Piezomagnetic Anisotropic Elastic Medium with an Elliptic Hole or Rigid Inclusion, Philos. Mag. Let., 72(6): 405-410.

$\mathrm{Hu}$, K.Q. and Li, G.Q. (2005). Constant Moving Crack in a Magnetoelectroelastic Material under Anti-plane Shear Loading, International Journal of Solids and Structures, 42(9-10): $2823-2835$.

Gao, C.F., Kessler, H. and Balke, H. (2003a). Crack Problems in Magnetoelectroelastic Solids. Part I: Exact Solution of a Crack, Inter. J. Eng. Sci., 41(9): 969-981.

Gao, C.F., Kessler, H. and Balke, H. (2003b). Crack Problems in Magnetoelectroelastic Solids. Part II: General Solution of Collinear Cracks, Int. J. Eng. Sci., 41(9): 983-994.

Gao, C.F., Tong, P. and Zhang, T.Y. (2004). Fracture Mechanics for a Mode III Crack in a Magnetoelectroelastic Solid, International Journal of Solids and Structures, 41(24-25): 6613-6629.

Li, X.F. (2003). Closed-form Solution for Two Collinear Mode-III Cracks in an Orthotropic Elastic Strip of Finite Width, Mechanics Research Communications, 30(4): 365-370.

Lin, J., Liu, Y. and Dean T.A. (2005). A Review on Damage Mechanisms, Models and Calibration Methods under Various Deformation Conditions, International Journal of Damage Mechanics, 14(4): 299-319.

Liu, J.X., Liu, X.L. and Zhao, Y.B. (2001). Green's Functions for Anisotropic Magnetoelectroelastic Solids with an Elliptical Cavity or a Crack, Inter. J. Eng. Sci., 39(12): 1405-1418.

Lu, J., Zhang, X. and Mai, Y.W. (2005). A Preliminary Study on Damage Wave in ElasticBrittle Materials, International Journal of Damage Mechanics, 14(2): 127-147.

Muskhelisvili, N.I. (1953). Singular Integral Equations, Noordhoff, Groningen.

Tian, W.Y. and Gabbert, U. (2005a). Macrocrack-microcrack Interaction Problem in Magnetoelectroelastic Solids, Mechanics of Materials, 37(5): 565-592.

Tian, W.Y. and Gabbert, U. (2005b). Parallel Crack Near the Interface of Magnetoelectroelastic Bimaterials, Computational Materials Science, 32(3-4): 562-567.

Voyadjis, G.Z. and Abu Al-Rub, R.K. (2006). A Finite Strain Plastic-damage Model for High Velocity Impacts using Combined Viscosity and Gradient Localization Limiters: Part II Numerical Aspects and Simulations, International Journal of Damage Mechanics, 15(4): 335-373.

Wang, B.L. and Mai, Y.W. (2003). Crack Tip. eld in Piezoelectric/Piezomagnetic Media, Euro. J. Mech. A/Solids, 22(4): 591-602.

Wang, B.L. and Mai, Y.W. (2004). Fracture of Piezoelectromagnetic Materials, Mechanics Research Communications, 31(1): 65-73.

Wang, X. and Shen, Y.P. (2002). The General Solution of Three-dimensional Problems in Magnetoelectroelastic Media, Inter. J. Eng. Sci., 40(10): 1069-1080.

Wei, Y., Chow, C.L., Vianco, P., et al. (2006). Isothermal Fatigue Damage Model for Lead-free Solder, International Journal of Damage Mechanics, 15(2): 109-119.

Ye, H., Basaran, C. and Hopkins, D.C. (2006). Experimental Damage Mechanics of Micro/ power Electronics Solder Joints under Electric Current Stresses, International Journal of Damage Mechanics, 15(1): 41-67. 